\title{
TENDÊNCIAS E CICLOS COMUNS ENTRE AS INFLAÇÕES DAS REGIÕES METROPOLITANAS DO BRASIL
}

\author{
Cristiano da Costa da Silva * \\ Nicolino Trompieri Neto ${ }^{\dagger}$ \\ IVAN CASTELAR $\ddagger$
}

\begin{abstract}
Resumo
O artigo utiliza a decomposição de tendências e ciclos comuns de Vahid \& Engle (1993) para verificar a presença de comovimentos de curto e longo prazo entre as taxas de inflação das regiões metropolitanas do Brasil, durante o período de outubro de 1995 até dezembro de 2014. Os testes comprovam a existência de duas tendências estocásticas comuns e seis ciclos comuns. Os resultados apontam inter-relações positivas de longo prazo entre a trajetória de inflação das regiões estudadas. As séries apresentaram também um comportamento similar de curto prazo, sendo caracterizadas como pró-cíclicas.
\end{abstract}

Palavras-chave: Inflação; Comovimentos; Ciclos Comuns; Tendências Comuns.

\begin{abstract}
This work uses the methodology implemented by Vahid \& Engle (1993) in order to investigate the presence of co-movements of short and longrun among the inflation rates of the metropolitan areas of Brazil during the 1995.9-2014.12 period. The tests have shown the existence of two common trends and six common cycles among the series. The results show that deviaton of long run balance in inflation rates of a given metropolitan region is able to influence the path of inflation of other metropolitan region. The series have a pro-cyclical behavior in the face of transitory shocks, sharing a similar short-term pattern.
\end{abstract}

Keywords: Inflation; Co-movements; Common Cycles; Common Trends JEL classification: E31, E32, C23

DOI: http://dx.doi .org/10.11606/1980-5330/ea128188

\footnotetext{
* Universidade Estadual do Rio Grande do Norte (UERN). Email:cristiano.dacostadasilva@hotmail.com

${ }^{\dagger}$ Universidade de Fortaleza (UNIFOR) e IPECE. E-mail: nicolino.trompieri@ipece.ce.gov.br

‡ Universidade Federal do Ceará (UFC). E-mail: lume1250@yahoo.com.br
} 


\section{Introdução}

Modelar a dinâmica inflacionária entre setores e regiões tem desempenhado um papel crucial nos estudos macroeconômicos. No contexto nacional, do ponto de vista de condução da política monetária, é fundamental investigar a susceptibilidade da natureza dos movimentos de curto prazo da taxa de inflação de uma dada região ser influenciada por características estruturais idiossincráticas de outras regiões (Gerard 2012, Gali \& Gertler 1999)

Uma vez que o Brasil é um país territorialmente extenso e possui dinâmicas regionais diferentes, a dinâmica da inflação pode não ser necessariamente similar entre as diferentes regiões do país. Dessa forma, as heterogeneidades regionais podem levar a demandas contraditórias sobre a condução da política monetária nacional. Logo, informações sobre a existência ou não de diferenças na dinâmica inflacionária, bem como em que medida essas disparidades surgem, são essenciais para o processo decisório dos policy makers (Beck et al. 2009). No fim do século XX, Gali \& Gertler (1999) apontavam que a discussão sobre a natureza dos ciclos econômicos da dinâmica inflacionária era amplamente debatida pela literatura, porém com poucas respostas empíricas disponíveis, esse quadro permanece estagnado pouco mais de uma década depois.

A teoria do ciclo real de negócios afirma que os ciclos são respostas naturais e eficientes da economia a alterações no nível de produção tecnológico disponível (Mankiw 1989); ou seja, choques permanentes. Já os Novos Keynesianos enfatizam o papel dos choques nominais e de demanda nos ciclos de negócios (Hairault \& Portier 1993); sendo estes distúrbios transitórios.

De acordo com as duas escolas, podemos particionar as flutuações que afetam o ciclo de negócios em choques permanentes (componente de tendência) e choques transitórios (componente de ciclo). Mills \& Holmes (1999) associam a componente de tendência a características estruturais e institucionais, enquanto o componente de ciclo é um fenômeno de curto prazo conectado às séries estacionárias.

O presente estudo tem por objetivo dar ênfase à análise do comovimento da dinâmica inflacionária entre determinadas regiões do Brasil por meio de diferentes fases de ciclos. A motivação disso é o maior peso que os agentes atribuem ao horizonte de curto prazo no processo decisório de condução de política monetária, tornando a volatilidade dos ciclos um fator importante para entender o ciclo de negócios.

Seguindo a metodologia de Vahid \& Engle (1993), será investigado se a taxa de inflação das regiões Sul, Sudeste e Nordeste do Brasil apresenta a mesma dinâmica de curto e longo prazo por meio da decomposição das séries em componente de tendência e componente de ciclo.

A extração de tendências e ciclos comuns para as séries temporais de dinâmica inflacionária possibilita avaliar qual o impacto de longo prazo de um aumento permanente na taxa de inflação de uma dada região metropolitana sobre as demais; quais as correlações existentes entre as flutuações de curto prazo; bem como se as tendências e ciclos individuais dos estados, causam no sentido de Granger, as tendências e ciclos individuais das demais regiões.

A proposta de investigar o comportamento da dinâmica temporal inflacionária em diferentes regiões perante aos choques econômicos é justificada pela importância da manutenção da estabilidade inflacionária para a economia brasileira. Um maior entendimento das relações entre as flutuações de 
curto prazo das taxas inflacionárias das regiões do Brasil pode contribuir para a condução de uma política monetária mais efetiva.

Com o intuito de atingir os objetivos propostos o artigo foi particionado em cinco seções: além dessa parte introdutória, em seguida, a Seção 2 apresenta a revisão de literatura. A Seção 3 apresenta o conjunto de técnicas econométricas necessárias para o alcance do objetivo proposto. A Seção 4 se dedica à apresentação da base de dados, bem como dos resultados obtidos, enquanto a Seção 5 traz as considerações finais.

\section{Referencial Teórico}

O maior nível de comercialização entre as regiões metropolitanas do Brasil alcançado nas últimas três décadas tornou a separação de variáveis entre componentes permanentes (tendências) e transitórios (ciclos) um tema relevante na pesquisa econômica. Entender o comportamento das regiões perante choques comuns ou a diferença na natureza dos seus comovimentos (tendências e ciclos comuns) é vital para a viabilidade das políticas públicas visando uma maior integração entre os estados.

A introdução de um procedimento geral para decompor séries de tempo que exibem homogeneidade não estacionária em componentes permanentes e transitórios remonta ao artigo seminal de Beveridge \& Nelson (1981), no qual a metodologia foi aplicada para mensurar e datar os ciclos de negócios da economia americana no pós-guerra.

A fim de investigar os comovimentos entre a taxa de crescimento do produto real em diferentes países, Vahid \& Engle (1993) formularam o que eles definiram como decomposição de Beveridge-Nelson-Stock-Watson para testar a presença de ciclos comuns. Essa decomposição revisa a especificação de cointegração para um quadro estrutural de séries temporais. Os autores utilizaram os conceitos de cointegração e correlação serial para extrair, respectivamente, tendências e ciclos comuns.

Cointegração refere-se à combinação linear que torna estacionária um conjunto de variáveis estocásticas, podendo existir $r$ vetores de cointegração independentes - os quais constituem o espaço de cointegração - dentro de um conjunto de $n$ variáveis. O número de tendências estocásticas comuns é determinado pela diferença entre o número de variáveis $(n)$ e o espaço de cointegração $(r)$. Assim, diz-se que existem $n-r$ relações de equilíbrio de longo prazo.

Uma proposição importante é que se existem $s$ combinações lineares linearmente independentes em um conjunto de $n$ variáveis integradas de Ordem $1-I(1)$-, que são passeios aleatórios, então essas variáveis devem compartilhar de $n-s$ ciclos comuns. Assim, a evidência de Serial Correlation Common Feature (SCCF) nas variáveis em primeira diferença implica na existência de ciclos comuns nas séries em nível. Um quadro no qual o número de vetores de cointegração $(r)$ mais o número de características comuns de correlação serial $(s)$ são iguais ao número de variáveis $(n)$ torna muito mais simples a extração dos componentes de ciclo e tendência.

Gutierrez \& Gomes (2009) aplicaram o método da decomposição multivariada Beveridge-Nelson-Stock-Watson para apurar o grau de comovimento dos ciclos de negócios dos países membros do Mercosul, os dados utilizados possuíam frequência anual de 1951 a 2003. O estudo confirmou a existência 
de tendências e ciclos comuns entre as economias analisadas. Adicionalmente, identificou-se evidências de comovimentos entre dois subgrupos - Brasil e Argentina; Paraguai e Uruguai.

Para examinar a dinâmica do Produto Interno Bruto de cinco países da América Latina, Hecq (2002) utilizou três modelos de ciclos comuns: Serial Correlation Common Feature (SCCF), Weak Form Reduce Post Structure (WF) e o Polynomial Serial Correlation Common Features (PSCCF). Foi encontrado que Brasil, Argentina, México, Peru e Chile dividem comovimentos no longo e curto prazo, sendo Argentina e Chile pró-cíclicos, enquanto Peru demonstrouse contra cíclico com respeito à Argentina e ao México.

Já Cerro \& Pineda (2002) utilizaram a abordagem de codependência para investigar os comovimentos no PIB Real entre onze países da América Latina ${ }^{1}$, usando dados trimestrais para o período entre 1960 até 2000. Testes indicaram a existência de sete tendências comuns e quatro ciclos comuns permitindo a decomposição entre componente cíclico e de tendência. Uma grande dispersão entre as correlações cíclicas foi encontrada entre os países analisados, com os países mais representativos (Brasil, México e Argentina) possuindo uma baixa correlação de relacionamento com os outros países da América Latina. Os autores apontaram, ainda, que os comovimentos cíclicos entre esses três países, que enfrentaram uma maior entrada e saída de capital, foram relativamente pequenos.

Já a decomposição de Beveridge \& Nelson (1981) e de Gonzalo \& Granger (1995) foram ambas examinadas em Carlino \& Sill (2001). Com dados trimestrais sobre a renda per capita das sete regiões principais dos Estados Unidos ${ }^{2}$ para o período de 1956 até 1995, o artigo apurou a dinâmica da tendência e do ciclo nessas regiões. Os autores apontaram que apesar de ambas as técnicas de decomposição possuir resultados semelhantes, em geral o fator comum baseado na decomposição de Gonzalo-Granger é tal que o componente permanente da decomposição, embora sendo um processo I(1), não é representável como um passeio aleatório multivariado.

Os resultados demonstraram que as sete regiões compartilham de ciclos e tendências comuns, e revelaram, também, a existência de uma considerável diferença na volatilidade dos ciclos entre as regiões, sendo o componente ciclo na região mais volátil (Sudeste) quase cinco vezes maior do que na região menos volátil (Extremo Oeste). Por fim, a análise da decomposição da variância apontou que o componente cíclico faz uma grande contribuição à previsão da variância da renda.

Muitos estudos centraram atenção na análise das flutuações em variáveis macroeconômicas - produto real, investimento e consumo - no período pósguerra nos EUA. Para alcançar tal objetivo diversas metodologias foram utilizadas, como o filtro de Hodrick-Prescott, o filtro band-pass e o método de extração de tendências e ciclos comuns, elaborado por Vahid \& Engle (1993).

Hodrick \& Prescott (1981) computaram o componente cíclico por meio de um procedimento de filtragem que extrai a tendência estocástica que varia suavemente ao longo do tempo. Um dos resultados encontrados foi um alto grau de variabilidade dos componentes agregados da demanda: os ciclos do investimento variam três vezes mais do que os ciclos do produto, enquanto esse

\footnotetext{
${ }^{1}$ Os países latinos americanos da amostra são Argentina, Bolívia, Brasil, Chile, Colômbia, Equador, México, Paraguai, Peru, Uruguai e Venezuela.

${ }^{2}$ As principais regiões dos Estados Unidos da amostra são Extremo Oeste, Grande Lagos, Meio Leste, Nova Inglaterra, Planícies, Sudeste e Sudoeste.
} 
último oscila duas vezes mais do que os ciclos do consumo. Uma das críticas tecidas ao filtro Hodrick-Prescott (HP) é a exigência, a priori, da determinação do parâmetro de suavidade do componente tendencial.

Já Baxter \& King (1999) desenvolveram uma aproximação ótima do filtro band-pass, o filtro é construído por meio de médias móveis de modo a isolar componentes periódicos de uma série de tempo que se encontram em uma determinada banda de frequência intermediária (ciclos), de forma a eliminar componentes de baixa frequência (tendência), assim como componentes de frequência muita alta (ruído). Os autores compararam uma série de méto$\operatorname{dos}^{3}$, e por fim se apurou que apesar do filtro HP ser em alguns casos uma aproximação razoável de um filtro ideal de ciclos de negócios, a aproximação ótima do filtro band-pass desenvolvida se mostrou mais flexível e de mais fácil implementação, produzindo uma melhor aproximação de um filtro ideal.

Issler \& Vahid (2001) utilizaram o método supracitado de Vahid \& Engle (1993) para averiguar se o produto per capita, consumo e investimento nos EUA compartilham ciclos comuns. Os resultados foram robustos à existência de ciclos comuns entre as variáveis. Adicionalmente, choques transitórios se mostraram fatores importantes para explicar a variação do produto e do investimento. Os autores concluíram que testes para a existência de ciclos comuns devem preceder estimações econométricas sempre que a presença da restrição de comovimentos de curto prazo entre as variáveis agregadas for provável. $\mathrm{O}$ ato de ignorar a existência de ciclos comuns leva a diferenças não triviais na importância relativa dos ciclos econômicos na análise do comportamento de variáveis agregadas.

Em uma linha metodológica semelhante à aplicada no presente trabalho, Manopimoke (2012) investigou o grau de importância do relacionamento com fatores externos da China e EUA na dinâmica dos preços experimentados em Hong Kong, por meio da técnica econométrica de componentes não observados. Utilizando dados trimestrais para o período entre 1986 até 2010, o autor foi capaz de capturar relações significativas entre a taxa de inflação de Hong Kong e distúrbios permanentes externos oriundos da China e EUA, com choques permanentes nos preços da China sendo importantes para explicar movimentos permanentes na tendência inflacionária de Hong Kong.

Por fim, Shaoping \& Xiaotao (2014) analisaram a hipótese de codependência entre os ciclos da inflação, dos índices de preços agrícolas e da condução da política monetária, por meio do agregado monetário M2, para a China. O artigo realizou a decomposição univariada de Beveridge-Nelson para examinar e decompor os ciclos codependentes das variáveis investigadas. Dentre os principais resultados foi verificado que os ciclos dos preços agrícolas precedem temporalmente a dinâmica de curto prazo da taxa de inflação. Outro fato digno de destaque é a forte codependência encontrada entre o ciclo inflacionário e o ciclo de política monetária, o que confirma a eficácia da estratégia de arrocho monetário como meio de contenção da taxa de inflação chinesa. Os dois resultados supracitados indicam que a aplicação de uma política moderada de expansão monetária, no intuito de promover crescimento econômico sustentável, deve ser condicionada a estabilidade dos níveis de preços agrícolas.

\footnotetext{
${ }^{3}$ Os autores utilizaram os seguintes métodos de filtragem: filtro first difference, filtro HodrickPrescott e filtro high-pass.
} 


\section{Modelo Econométrico}

\subsection{Teste de Raiz Unitária}

Em relação aos testes de estacionariedade empregados no presente trabalho, são utilizados os testes de Dickey-Fuller Aumentado (ADF) e o método proposto por Elliott et al. (1992), para verificar se as séries que compõem o modelo são estacionárias. Contudo, devido à provável existência de quebra na tendência observada nos dados, faz-se uso de dois testes adicionais para verificar a ocorrência de raiz unitária com quebra estrutural. Os testes aplicados para verificar a existência de raiz unitária com quebra estrutural, são os testes propostos por Zivot \& Andrews (1992) e uma modificação do teste ADF permitindo uma única quebra estrutural, formulado a partir dos trabalhos de Perron (1989), Perron \& Vogelsang (1992), Vogelsang \& Perron (1998) e Banerjee et al. (1992).

\subsection{Modelo Econométrico}

Para avaliar a hipótese de que as taxas de inflação das principais regiões metropolitanas do Brasil possuem relações de equilíbrio tanto no longo quanto no curto prazo, o presente estudo seguirá a abordagem de decomposição multivariada de Beveridge-Nelson-Stock-Watson (BNSW) apresentada em Vahid \& Engle (1993).

A decomposição BNSW testa a existência de dinâmicas comuns de longo e curto prazo sobre um modelo VAR estimado. Inicialmente, considere que as taxas de inflação das $n$ regiões metropolitanas sejam representados por um Vetor Auto Regressivo (VAR) de ordem finita $p$.

$$
Y_{t}=\varphi_{1} Y_{t-1}+\varphi_{2} Y_{t-2}+\ldots+\varphi_{p} Y_{t-p}+\varepsilon_{t}
$$

em que $Y_{t}$ representa um vetor de $n$ séries integradas de primeira Ordem, $I(1), \varphi_{i}, i=1,2, \ldots, p$ são matrizes de dimensão nxne $\varepsilon_{t} \sim \operatorname{Normal}(0, \Omega)$, $E\left(\varepsilon_{t}\right)=0$ e $E\left(\varepsilon_{t} \varepsilon_{\tau}\right)=\left\{\Omega\right.$, set $=\tau \mathrm{e} 0_{n} x n$, se $\left.t \neq \tau\right\}$; em que $\Omega$ é não singular. Dessa forma, o Modelo (1) pode ser escrito de forma equivalente como:

$$
\Pi\left(L_{t}\right) Y_{t}=\varepsilon_{t}
$$

em que $\Pi\left(L_{t}\right)=I_{n}-\sum_{i=1}^{p} \varphi_{i} L^{i}$, e $L$ representa o operador defasagem. Note que se $L=1$, então $\Pi(1)=I_{n}-\sum_{i=1}^{p} \varphi_{i}$.

\subsection{Restrições de Longo Prazo - Cointegração}

Assuma as seguintes hipóteses:

Proposição 1: A matriz $\Pi($.$) satisfaz:$

1) Posto $(\Pi(1))=r, 0<r<n$, tal que $\Pi(1)$ pode ser expresso como $\Pi(1)=$ $-\alpha \beta^{\prime}$, em que $\alpha$ e $\beta$ são matrizes (nxr) com posto cheio $r$.

2) A equação característica $|\Pi(L)|=0$ possui $n-r$ raízes iguais a 1 e todas as outras fora do círculo unitário.

A hipótese 1 implica que $Y_{t}$ é uma série cointegrada de Ordem $(1,1)$. Os elementos de $\alpha$ representam os coeficientes de ajuste de velocidade ao equilíbrio de longo prazo e $\beta$ o espaço de cointegração. Decompondo a matriz polinomial $\Pi(L)=\Pi(1) L+\Pi^{*}(L) \Delta$, em que $\Delta=(1-L)$ denota o operador de diferença, pode-se obter um modelo de vetor de correção de erros (VEC): 


$$
\Delta Y_{t}=\alpha \beta^{\prime} Y_{t-1}+\sum_{i=1}^{p} \Gamma_{j} \Delta Y_{t-j}+\varepsilon_{t}
$$

em que, $\alpha \beta^{\prime}=-\Pi(1), \Gamma_{j}=-\sum_{k=j+1}^{p} \theta_{k}(j=1,2, \ldots, p-1)$ e $\Gamma_{0}=I_{n}$.

Assim como na análise dos testes de raiz unitária, a não incorporação de quebras estruturais à abordagem de cointegração tende a enviesar os resultados encontrados, de forma a não rejeitar a hipótese nula de inexistência de cointegração, quando a teoria prediz que a mesma deve existir. Portanto, será aplicado o teste desenvolvido por Johansen et al. (2000), no qual a distribuição assintótica difere do usual "teste do traço" de Johansen (1988) por utilizar variáveis dummies que identificam quebras estruturais na tendência dos vetores cointegrantes. Assim, o teste de Johansen et al. (2000) fornece informações que permitem calcular os valores críticos de forma mais adequada (Giles \& Godwin 2012).

\subsection{Restrições de Curto Prazo - Característica de Correlação Serial Comum}

O modelo $\operatorname{VAR}(p)$ pode possuir restrições de curto prazo conforme demonstrado por Vahid \& Engle (1993).

Definição 1: A Equação (4) apresentará característica de correlação serial comum (SCCF) se existir uma matriz checkbeta $a_{n}^{\prime} x s$ de posto $s$, representando o espaço de cocaracterização, tal que $\tilde{\beta}^{\prime} \Delta Y_{t}=\tilde{\beta}^{\prime} \varepsilon_{t}$, em que $\tilde{\beta}^{\prime} \varepsilon_{t}$ é um vetor de dimensão $s$ que é uma inovação em relação a toda informação anterior ao período $t$.

Consequentemente existirão restrições de características de correlação serial comum se as seguintes condições forem satisfeitas:

$$
\begin{aligned}
& \text { Proposição 2: } \tilde{(} \beta)^{\prime} \Gamma=0_{s} x n \quad \forall i=1, \ldots, p-1 \\
& \text { Proposição 3: } \left.\tilde{(} \alpha)^{\prime} \beta \tilde{(} \alpha\right)^{\prime}=0_{s} x n
\end{aligned}
$$

\subsection{Decomposição Tendência-Ciclo}

A decomposição tendência-ciclo BNSW pode ser introduzido por meio da representação de Wold do vetor estacionário $\Delta Y_{t}$ dado por:

$$
\Delta Y_{t}=C(L) \varepsilon_{t}
$$

em que $\mathrm{C}(\mathrm{L})=\sum_{i=0}^{\infty} C_{i} L^{i}$ é uma matriz polinomial no operador defasagem, $C_{0}=I_{n}$ e $\sum_{i=0}^{\infty} i\left|C_{i}\right|<\infty$. Usando a seguinte fatoração polinomial $C(L)=C(1)+$ $\Delta C^{*}(L)$, é possível decompor $\Delta Y_{t}$ como:

$$
\Delta Y_{t}=C(1) \varepsilon_{t}+\Delta C^{*}(L) \varepsilon_{t}
$$

em que $C_{i}^{*}=\sum_{j>i}^{\infty}\left(-C_{j}\right), i \geq 0$, e $C_{0}^{*}=I_{n}-C(1)$. Ignorando os valores iniciais $Y_{0}$ e integrando ambos os lados de (5), obtêm-se:

$$
Y_{t}=C(1) \sum_{j=1}^{T} \varepsilon_{t}+C^{*}(L) \varepsilon_{t}=T_{t}+C_{t}
$$


A Equação (7) representa a decomposição BNSW em que $Y_{t}$ é decomposto em $1^{n}$ j processos de passeio aleatório - tendências estocásticas - e inj processos estacionários - ciclos. Assim, $T_{t}=C(1) \sum_{j=1}^{T} \varepsilon_{t}$ e $C_{t}=C^{*}(L) \varepsilon_{t}$ representam o componente de tendência e ciclo, respectivamente. Se as restrições de longo prazo forem verificadas, então os $r$ vetores de cointegração eliminam o componente de tendência estocástica o que implica que $\beta^{\prime} C(1)=0$.

Desse modo $C(1)$ tem dimensão $n-r$, o que implica na existência de $n-r$ tendências comuns. Analogamente, sob as restrições de curto prazo, existem $s$ vetores de cocaracterização que eliminam os ciclos, $\tilde{(} \beta)^{\prime} C^{*}(L)=0$, e $C^{*}(L)$ possui dimensão $n-s$, o qual é o número de ciclos comuns.

Uma vez que os vetores de cointegração e cocaracterização são linearmente independentes (Vahid \& Engle 1993) o número de vetores de cointegração adicionado ao de vetores de cocaracterização deve ser menor ou igual ao número de variáveis $-r+s \leq n$.

Para obter as tendências comuns basta pré-multiplicar a Equação (7) por $(\beta)^{\prime}$, de tal forma:

$$
\tilde{\beta}^{\prime} Y_{t}=\tilde{\beta}^{\prime} C(1) \sum_{j=1}^{T} \varepsilon_{t}=\tilde{\beta}^{\prime} T_{t}
$$

Essa combinação linear não contem ciclos porque os vetores cocaracterísticos os eliminam. Da mesma forma, para obter os ciclos comuns é necessário e suficiente pré-multiplicar a Equação (7) por $\beta^{\prime}$ :

$$
\beta^{\prime} Y_{t}=\beta^{\prime} C(1) C^{*}(L) \varepsilon_{t}=\beta^{\prime} C_{t}
$$

Essa combinação linear não contem componentes de tendência, porque as mesmas são eliminadas pelos vetores de cointegração. Um caso especial emerge quando $r+s=n$. Nesse caso, é extremamente simples estimar os componentes de tendência e ciclo de $Y_{t}$. Uma vez que $\tilde{\beta}^{\prime}$ e $\beta^{\prime}$ são matrizes linearmente independentes, pode-se construir uma matriz $A$, tal que $A_{n} x n=\left(\tilde{\beta}^{\prime}, \beta^{\prime}\right)^{\prime}$ possui posto completo, sendo inversível. Note que a matriz inversa pode ser particionada como $A^{-1}=\tilde{\beta}^{-}, \beta^{-}$e os componentes de tendência e ciclo podem ser obtidos como a seguir:

$$
Y_{t}=A^{-1} A Y_{t}=\tilde{\beta}^{-}\left(\tilde{\beta}^{\prime} Y_{t}\right)+\beta^{-}\left(\beta^{\prime} Y_{t}\right)=T_{t}+C_{t}
$$

em que $T_{t}=\tilde{\beta}^{-}\left(\tilde{\beta}^{\prime} Y_{t}\right)$ e $C_{t}=\beta^{-}\left(\beta^{\prime} Y_{t}\right)$. Portanto, tendência e ciclo são combinações lineares de $Y_{t}$. Note que $T_{t}$ é gerado por uma combinação linear de $Y_{t}$ usando os vetores cocaracterísticos, contendo o componente de longo prazo (visto que $\tilde{\beta}^{\prime} Y_{t}$ é um componente que segue um passeio aleatório). Por outro lado, $C_{t}$ é gerado por uma combinação linear de $Y_{t}$ usando os vetores de cointegração, contendo o componente de curto prazo (porque $\beta^{\prime} Y_{t}$ é $I(0)$ e serialmente correlacionado).

\subsection{Teste de Ciclos Comuns}

A existência de ciclos comuns será testada a partir do procedimento de correlações canônicas delineado por Vahid \& Engle (1993). Conforme Roache (2008), o objetivo do teste é encontrar combinações lineares que removam correlações baseadas em um conjunto de valores do passado. Sendo essas combinações lineares definidas como vetores cocaracterísticos. 
O primeiro passo é estimar o VEC para descobrir os termos de correção erro da série, ou seja, para conhecer as relações de longo prazo. Retomando a Equação (4):

$$
\Delta Y_{t}=\alpha \beta^{\prime} Y_{t-1}+\sum_{i=1}^{p} \Gamma_{j} \Delta Y_{t-j}+\varepsilon_{t}
$$

Definindo $\rho_{t}$ como um vetor de dimensão $(n x 1)$ obtido como combinação linear do vetor $\delta Y_{t}$ e $\eta_{t}$ como o vetor de dimensão $\left((n p+r) x_{1}\right)$ obtido como combinação linear das defasagens de $\delta Y_{t}$ e dos termos de correção de erro $\left(x_{t}\right)$.

$$
\begin{array}{r}
p_{t}=A^{\prime} \Delta Y_{t} \\
\eta_{t}=B^{\prime}\left[\Delta Y_{t} \ldots \Delta Y_{t-p+1} \alpha Y_{t-1}\right]
\end{array}
$$

As matrizes $A_{n x n}$ e $B_{n x(n p+r)}$ são escolhidas de modo que quatro condições sejam satisfeitas. As duas primeiras afirmam que os elementos de $\rho_{t}$ e $\eta_{t}$ possuem variância unitária. A terceira condição assegura que o $i$-ésimo elemento de $\rho_{t}$ seja não correlacionado com o $j$-ésimo elemento de $\eta_{t}$, e a condição final condiciona a ordenação dos elementos de $\rho_{t}$ e $\eta_{t}$ de modo que:

$$
1 \geq \lambda_{1} \geq \ldots \geq \lambda_{n} \geq 0
$$

As correlações canônicas e os valores de A e B podem ser calculados a partir da matriz de covariância de $\Delta Y_{t}$ e $x_{t}$ por meio dos autovalores e autovetores. O teste estatístico é análogo ao teste do traço do procedimento de Johansen (1988), com a hipótese nula sendo que o espaço cocaracterístico é pelo menos de dimensão $s$, é calculado como:

$$
C(p, s)=-(T-p-1) \sum_{i=1}^{s} \log \left(1-\lambda_{i}^{2}\right)
$$

em que $\lambda_{i}^{2}$ são as menores $s$ correlações canônicas elevadas ao quadrado. Sob a hipótese nula $C(p, s)$ segue uma distribuição $\chi^{2} \operatorname{com} s(n p+r)-s(n-s)$ graus de liberdade, em que $n$ é a dimensão do sistema.

\section{Estimação Empírica e Base de Dados}

\subsection{Base de Dados}

Diante do objetivo proposto, o presente estudo faz uso do Índice Nacional de Preços ao Consumidor Amplo - IPCA - disponibilizado pelo Instituto Brasileiro de Geografia e Estatística (IBGE). O IPCA é calculado de forma contínua e sistemática para as regiões metropolitanas de Belém, Belo Horizonte, $\mathrm{Cu}$ ritiba, Fortaleza, Porto Alegre, Recife, Rio de Janeiro, Salvador e São Paulo. Logo a pesquisa abrange um número restrito de regiões do Brasil.

Frente a essa limitação, sugere-se aqui analisar a presença de tendências e ciclos comuns para as regiões metropolitanas pesquisadas do Sul, Sudeste e Nordeste: 
Tabela 1: Descrição das regiões metropolitanas contempladas na pesquisa

\begin{tabular}{lccc}
\hline Região Metropolitana & Código & Estado & Região do Brasil \\
\hline Belo Horizonte & BH & Minas Gerais & Sudeste \\
Curitiba & CUR & Paraná & Sul \\
Fortaleza & FOR & Ceará & Nordeste \\
Porto Alegre & POA & Rio Grande do Sul & Sul \\
Recife & REC & Pernambuco & Nordeste \\
Rio de Janeiro & RJ & Rio de Janeiro & Sudeste \\
Salvador & SAL & Bahia & Nordeste \\
São Paulo & SP & São Paulo & Sudeste \\
\hline
\end{tabular}

Fonte: Elaborado pelo autor.

Os dados, em formato de variação percentual mensal, são homogêneos no que se refere ao período amostral abrangido, sendo janeiro de 1980 a data inicial da pesquisa. No intuito de evitar a época de forte instabilidade econômica a nível nacional, o presente estudo fez a opção de utilizar somente as observações amostrais para o período pós Plano Real, compreendendo o período de outubro de 1995 até dezembro de 2014, abrangendo uma amostra de 232 observações para cada variável de interesse.

Cabe destacar, adicionalmente, que as séries históricas analisadas foram construídas em termos de variações acumuladas em 12 meses. A utilização de tal métrica justifica-se por dois motivos distintos: primeiro pelo fato de que o sistema de metas de inflação utilizadas pelo Governo Federal é acompanhado em termos anuais; e por fim, a métrica ajusta a sazonalidade naturalmente, não sendo necessário, portanto, a aplicação de filtros de ajuste sazonal, os quais tendem a afetar os resultados da decomposição de tendências e ciclos comuns - para detalhes adicionais vide Hecq (2002).

A Figura 1 reporta a trajetória temporal das oito séries durante o período estudado, em termos de variação acumulada. Por meio da inspeção visual observa-se uma grande proximidade na trajetória de todas as séries. Analisando a trajetória comum das regiões metropolitanas é possível notar uma tendência de forte queda na variação anual acumulada do índice de preços até o final de 1998. A partir de 1998 há um movimento inverso na trajetória, com tendência de crescimento moderado da taxa de inflação até meados de 1999, e uma posterior estabilidade até o final de 2002.

Já no período entre o último trimestre de 2002 ao trimestre inicial de 2004 é nítida a presença de um comportamento atípico na evolução das oito séries, com um crescimento repentino e uma posterior queda acentuada na taxa de variação anual acumulada de inflação, voltando a um nível similar do período anterior ao final de 2002.

Deste período em diante verifica-se a presença de movimentos oscilatórios em torno da média na taxa de inflação, denotando um período de estabilidade no índice, mesmo frente ao período de crise internacional instaurada a partir de setembro de 2008, reflexo da política monetária para contenção da inflação, como a elevação da taxa de juros.

A Tabela 2 reproduz algumas das principais estatísticas descritivas consoantes as variáveis analisadas. Ao longo do período amostral todas as regiões metropolitanas experimentaram taxas médias de inflação anualizadas semelhantes. O Rio de Janeiro possui a maior taxa média com $7,45 \%$ enquanto Fortaleza possui a menor taxa com $6,85 \%$. 


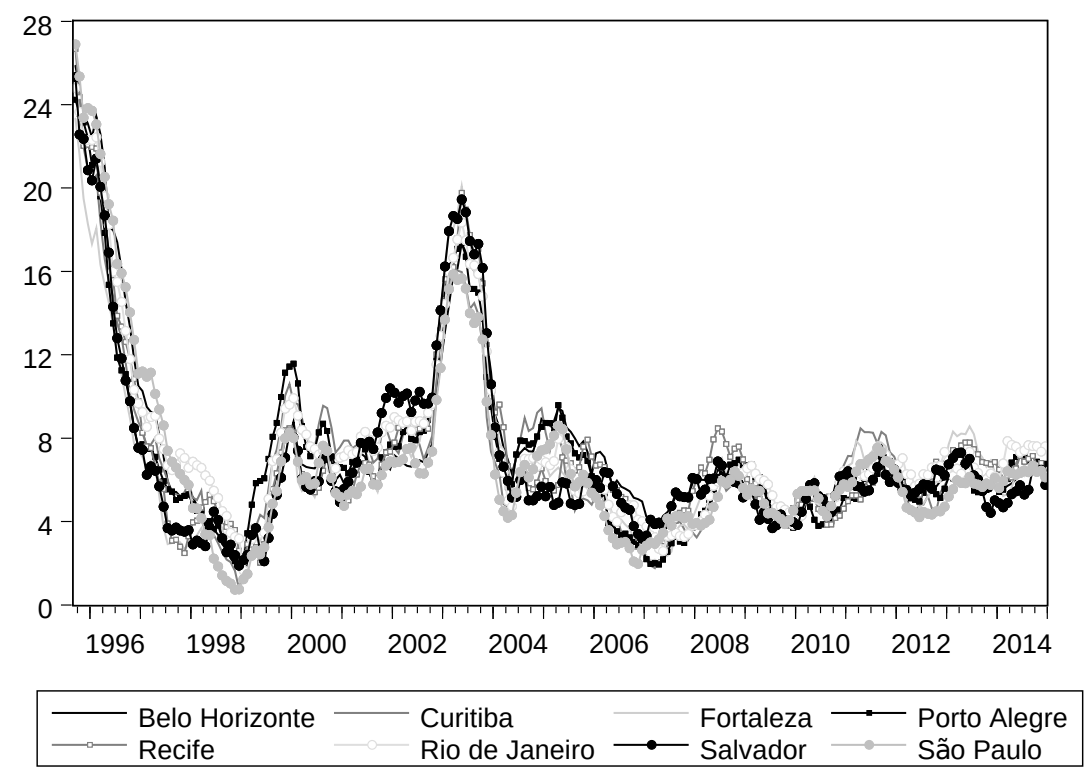

Obs: Taxa de variação acumulada anualmente, durante o período de outubro de 1995 até dezembro de 2014, totalizando 232 observações. Fonte: Elaborado pelo autor.

Figura 1: Trajetória temporal da taxa de inflação

Tabela 2: Estatísticas descritivas das taxas de inflação das regiões metropolitanas

\begin{tabular}{lcccccccc}
\hline Métrica & BH & CUR & FOR & POA & \multicolumn{1}{c}{ REC } & RJ & \multicolumn{1}{c}{ SAL } & SP \\
\hline Média & $7,45 \%$ & $7,09 \%$ & $6,85 \%$ & $7,16 \%$ & $7,25 \%$ & $7,58 \%$ & $7,00 \%$ & $6,86 \%$ \\
Máximo & $25,87 \%$ & $24,66 \%$ & $23,33 \%$ & $24,23 \%$ & $26,66 \%$ & $24,79 \%$ & $25,32 \%$ & $26,89 \%$ \\
Mínimo & $1,26 \%$ & $0,78 \%$ & $2,05 \%$ & $1,92 \%$ & $2,03 \%$ & $1,66 \%$ & $1,89 \%$ & $0,74 \%$ \\
Desvio Padrão & $4,26 \%$ & $3,93 \%$ & $3,86 \%$ & $4,04 \%$ & $4,24 \%$ & $4,10 \%$ & $4,26 \%$ & $4,46 \%$ \\
Assimetria & 2,30 & 1,91 & 2,01 & 2,04 & 2,21 & 2,12 & 2,15 & 2,26 \\
Curtose & 8,51 & 7,69 & 7,13 & 7,80 & 8,05 & 7,73 & 7,34 & 8,65 \\
\hline
\end{tabular}

Estatísticas obtidas a partir das séries mensais que compõem o acervo do Índice de Preços ao Consumidor Amplo (IPCA) durante o período de novembro de 1995 até dezembro de 2014.

Fonte: IBGE.

O índice de São Paulo é robustamente mais volátil, possuindo a taxa máxima de inflação mais alta, 26,89\%, além da menor taxa mínima de variação acumulada de inflação, $0,74 \%$. Consoante às outras métricas, os índices de assimetria apontam na mesma direção e todas as variáveis estudadas são leptocúrticas; ou seja, as distribuições apresentam uma curva de frequência mais fechada que a normal.

\subsection{Testes de Estacionariedade}

Inicialmente foram feitos, em caráter preliminar, testes de raiz unitária das quais as estatísticas não levam em conta a presença de quebra estrutural. Conforme pode ser notado na Tabela 3, os resultados apontam para a presença de não estacionariedade em todas as séries em nível. Duas exceções, porém, ocorrem de acordo com o teste ADF, com as séries de Recife e São Paulo sendo consideradas estacionárias em nível a um nível de 5\% de significância. 
Uma das condições necessárias para que a decomposição em tendências e ciclos sugerida por Vahid \& Engle (1993) seja válida é que todas as séries sejam integradas de Ordem 1. Com base em ambos os testes propostos os oito índices apresentam natureza estacionária em $1^{\text {a }}$ diferença. Portanto, a presença de não estacionariedade em nível sugere a existência de tendência estocástica.

Porém, como já fora discutido na Seção 4.1, as séries experimentaram momentos de mudança repentina de comportamento durante o período final de 2002 até o primeiro trimestre de 2004; esse comportamento indica a provável presença de quebras estruturais na trajetória temporal das variáveis estudadas. Portanto, para que os resultados dos testes de raiz unitária sejam robustos a presença de mudanças repentinas de comportamento é necessário utilizar métricas que adicionem uma dummy para captar o efeito de quebras estruturais nos testes de raiz unitária.

Ao incorporar a presença endógena de quebra estrutural nos testes de estacionariedade, todas as séries - a exceção de Curitiba a um nível de 5\%, segundo o teste de Zivot \& Andrews (1992) - se apresentaram não estacionárias em nível, mas estacionárias em $1^{\text {a }}$ diferença. Logo, os resultados indicam que a taxa de variação acumulada anualmente de inflação das oito regiões metropolitanas estudadas seguem um processo integrado de $1^{\mathrm{a}}$ ordem.

\subsection{Teste de Cointegração}

Conforme os resultados encontrados na Seção 4.2, as séries apresentam a presença de quebra estrutural e uma tendência estocástica, sendo integradas de Ordem 1. Visando lidar com a presença de quebra estrutural nas séries, foi implementado o teste de Chow, cujo tem por objetivo testar a igualdade de um conjunto de coeficientes entre duas regressões a partir da estatística $\mathrm{F}$, para testar a hipótese nula de quebra estrutural no período de outubro de 2003 um ano após a eleição do Presidente Luis Inácio Lula da Silva. A estatística de Chow não rejeita a hipótese nula de mudança estrutural no período de outubro de 2003 nas estimativas, havendo instabilidade no modelo no período anterior a essa data e estabilidade logo após.

A partir daí é possível estimar as relações de equilíbrio de longo prazo entre as variáveis por meio do teste de cointegração multivariado de Johansen et al. (2000), o qual incorpora uma dummy associada a quebra estrutural identificada em outubro de 2003 na determinação de seus valores críticos, a especificação considera também intercepto e tendência restrita no nível.

Inicialmente, a ordem de defasagem foi definida de acordo com o critério de informação de Schwarz, cujo indicou uma defasagem ${ }^{4}$. No entanto, observou-se a presença de correlação serial nos resíduos do VAR estimado. Athanasopoulos et al. (2011) enfatizam que os modelos tradicionais de seleção de defasagens tendem a subestimar o verdadeiro número de defasagens em modelos VAR na presença de restrições de longo e curto prazo. Diante disso, reestimou-se o modelo utilizando doze lags (ordem selecionada pelo critério de Akaike), os testes de especificação realizados sobre os resíduos estimados indicaram ausência de correlação serial, o que assegura a adequabilidade do modelo escolhido.

\footnotetext{
${ }^{4}$ Os resultados dos critérios de informação e dos testes de correlação serial encontram-se em anexo.
} 
Tabela 3: Testes de estacionariedade não incorporando quebra estrutural

\begin{tabular}{|c|c|c|c|c|c|c|c|c|c|}
\hline & & BH & CUR & FOR & POA & REC & RJ & SAL & SP \\
\hline \multirow{3}{*}{$\begin{array}{l}\mathrm{ADF}(\mathrm{b}) \\
\left(\mathrm{H}_{0}: \text { Série com raiz unitária }\right)\end{array}$} & & & & & & & & & \\
\hline & Teste em Nível & $\begin{array}{c}-3.33^{*} \\
(0,06)\end{array}$ & $\begin{array}{r}-2,85 \\
(0,18)\end{array}$ & $\begin{array}{r}-2,84 \\
(0,18)\end{array}$ & $\begin{array}{r}-2,92 \\
(0,16)\end{array}$ & $\begin{array}{c}-3,52^{* *} \\
(0,05)\end{array}$ & $\begin{array}{r}-2,81 \\
(0,19)\end{array}$ & $\begin{array}{r}-2,94 \\
(0,15)\end{array}$ & $\begin{array}{c}-3,46^{* *} \\
(0,05)\end{array}$ \\
\hline & Teste na $1^{a}$ Diferença & $\begin{array}{c}-4,84^{* * * *} \\
(0,00)\end{array}$ & $\begin{array}{c}-4,48^{* * * *} \\
(0,00)\end{array}$ & $\begin{array}{c}-4,80^{* * * *} \\
(0,00)\end{array}$ & $\begin{array}{c}-4,85^{* * *} \\
(0,00)\end{array}$ & $\begin{array}{c}-4,14^{* * * *} \\
(0,00)\end{array}$ & $\begin{array}{c}-5,04^{* * * *} \\
(0,00)\end{array}$ & $\begin{array}{c}-4,76^{* * *} \\
(0,00)\end{array}$ & $\begin{array}{c}-4,75^{* * *} \\
(0,00)\end{array}$ \\
\hline $\begin{array}{l}\text { Elliott et al. }(1996)(\mathrm{c}) \\
\left(\mathrm{H}_{0} \text { : Série com raiz unitária }\right)\end{array}$ & $\begin{array}{c}\text { Teste em Nível } \\
\text { Teste na } 1^{\text {a }} \text { Diferença }\end{array}$ & $\begin{array}{l}27,65 \\
1,51^{* * *}\end{array}$ & $\begin{array}{l}40,60 \\
1,06^{* * *}\end{array}$ & $\begin{array}{l}24,92 \\
0,01^{* * *}\end{array}$ & $\begin{array}{l}26,04 \\
0,00^{* * *}\end{array}$ & $\begin{array}{l}17,25 \\
2,19^{* * *}\end{array}$ & $\begin{array}{l}41,10 \\
0,18^{* * *}\end{array}$ & $\begin{array}{l}19,02 \\
0,42^{* * * *}\end{array}$ & $\begin{array}{c}43,28 \\
0,08^{* * * *}\end{array}$ \\
\hline
\end{tabular}

Teste de estacionariedade aplicado às séries mensais acumuladas a taxa anual para as regiões metropolitanas do Brasil durante o período de outubro de 1995 até dezembro de 2014. Todos os testes foram feitos levando em conta a presença de um intercepto e uma tendência. Fonte: IBGE. (b) Estatística $t$ reportada com o respectivo p-valor entre parênteses. Escolha de defasagens, segundo o critério de Akaike. (c)

Estatística $P$ reportada. Escolha de defasagens, segundo o critério de Akaike. ${ }^{*}$ Rejeição da hipótese nula a $10 \% .{ }^{* *}$ Rejeição da hipótese nula a

$5 \%$. ${ }^{* * *}$ Rejeição da hipótese nula a $1 \%$. 
Tabela 4: Testes de estacionariedade incorporando quebra estrutural

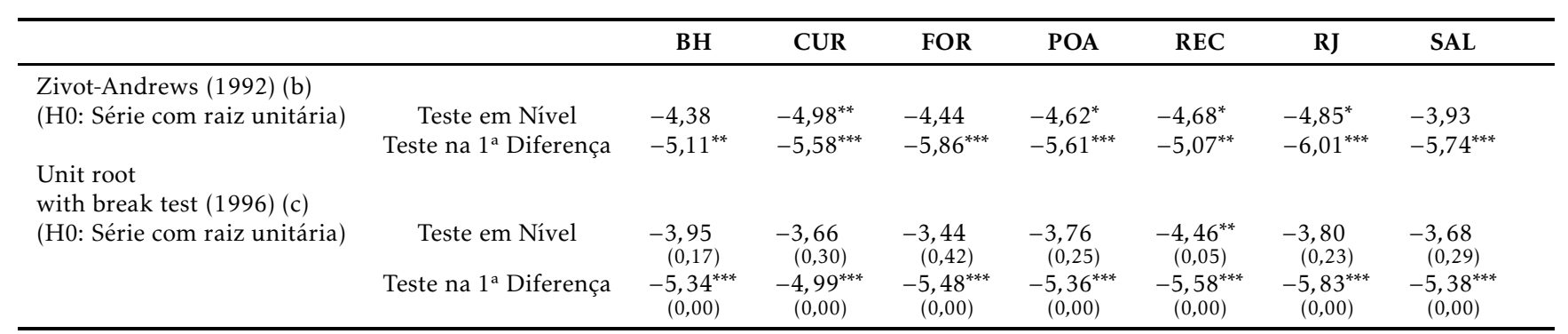

Teste de estacionariedade incorporando quebras estruturais endógenas aplicadas as séries mensais acumuladas a taxa anual para as

regiões metropolitanas do Brasil durante o período de novembro de 1995 até dezembro de 2014. Todos os testes foram feitos levando em

conta a presença de um intercepto e uma tendência. Fonte: IBGE. (b) Estatística $t$ reportada. Escolha de defasagens, segundo o critério de Schwarz, com limitação máxima de 12 defasagens. (c) Estatística $t$ reportada com o respectivo p-valor entre parênteses. Escolha de

defasagens, segundo o critério de Akaike. ${ }^{*}$ Rejeição da hipótese nula a $10 \% .{ }^{* *}$ Rejeição da hipótese nula a 5\%. ${ }^{* * *}$ Rejeição da hipótese nula a $1 \%$. 
Tabela 5: Teste de cointegração de Johansen et. al(2000)

\begin{tabular}{lccc}
\hline $\mathbf{N}^{\mathbf{0}}$ de Vetores & Estatística do Traço & Valores Críticos a 5\% & p-valor \\
\hline $\mathrm{r}=0$ & 483,95 & 195,21 & 0,0000 \\
$r \leq 1$ & 306,94 & 151,78 & 0,0000 \\
$r \leq 2$ & 216,03 & 118,77 & 0,0000 \\
$r \leq 3$ & 154,66 & 89,66 & 0,0000 \\
$r \leq 4$ & 97,64 & 64,46 & 0,0000 \\
$r \leq 5$ & 60,80 & 43,19 & 0,0010 \\
$r \leq 6$ & 28,65 & 25,72 & 0,0452 \\
$r \leq 7$ & 8,93 & 12,02 & 0,2774 \\
\hline \multicolumn{4}{l}{ Teste de cointegração aplicado às séries mensais acumuladas a taxa anual } \\
para as regiões metropolitanas do Brasil durante o período de outubro de \\
1995 até dezembro de 2014. O teste inclui uma dummy referente à \\
quebra estrutural na data de 2003-2009 e utiliza uma defasagem de \\
acordo com critério de Schwarz, considerando-se a presença de \\
intercepto e tendência. Os valores críticos foram obtidos de acordo em \\
Johansen et. al(2000).
\end{tabular}

O resultado do teste do traço de Johansen et al. (2000) - Tabela 5 - aponta a presença de sete vetores cointegrantes, a um nível de 5\% de significância. Consequentemente existem uma única relação de equilíbrio de longo prazo comum entre as séries (visto que o número de tendências comuns é dado pela diferença entre o número de variáveis $(n=8)$ e o espaço de cointegração $(r=$ 7)) e no máximo sete ciclos comuns.

Assim, a dinâmica temporal da taxa de inflação das séries analisadas é determinada por um componente agregado comum e por características individuais presentes em cada região metropolitana.

Adicionalmente, o procedimento de Johansen et al. (2000) permite normalizar o vetor de cointegração, possibilitando, assim, identificar as relações econômicas das séries em termos de elasticidade. A normalização foi feita de tal forma que as $r$ primeiras séries no vetor $x_{t}$ formam uma matriz identidade.

Tabela 6: Vetor de cointegração normalizado

\begin{tabular}{lllllllc}
\hline BH & CUR & FOR & POA & REC & RJ & SAL & SP \\
\hline 1,000 & 0,000 & 0,000 & 0,000 & 0,000 & 0,000 & 0,000 & $-0,869$ \\
0,000 & 1,000 & 0,000 & 0,000 & 0,000 & 0,000 & 0,000 & $-0,947$ \\
0,000 & 0,000 & 1,000 & 0,000 & 0,000 & 0,000 & 0,000 & $-0,952$ \\
0,000 & 0,000 & 0,000 & 1,000 & 0,000 & 0,000 & 0,000 & $-0,718$ \\
0,000 & 0,000 & 0,000 & 0,000 & 1,000 & 0,000 & 0,000 & $-0,779$ \\
0,000 & 0,000 & 0,000 & 0,000 & 0,000 & 1,000 & 0,000 & $-0,850$ \\
0,000 & 0,000 & 0,000 & 0,000 & 0,000 & 0,000 & 1,000 & $-1,159$ \\
\hline
\end{tabular}

Vetor de cointegração aplicado as séries mensais acumuladas à taxa anual para as regiões metropolitanas do Brasil durante o período de outubro de 1995 até dezembro de 2014. O VEC foi estimado levando em conta a presença de intercepto e tendência, e utiliza doze lags de acordo com o critério de Akaike.

Em termos econômicos, as elasticidades de longo prazo estimadas apresentaram-se aproximadamente unitárias, o que indica forte similaridade no comportamento das inflações regionais frente a choques permanentes no sistema. 


\subsection{Teste de Ciclos Comuns}

Uma vez confirmada a hipótese de cointegração, o passo seguinte é analisar a existência de ciclos comuns. Seguindo a metodologia de Vahid \& Engle (1993) exposta na Seção 3.5, o número de vetores de cointegração mais o número de vetores de cocaracterização não podem exceder o número de variáveis no sistema $(r+s \leq n)$. Visto que existem sete vetores de cointegração $(r=7)$ entre as oito regiões metropolitanas $(n=8)$, deve existir, então, no máximo um vetor de cocaracterização $(s \leq 1)$, o que implica na ocorrência de sete ciclos comuns.

Utilizando o teste desenvolvido por Warne (2008), que seleciona automaticamente o número máximo de ciclos, foi verificado que a hipótese da existência de sete ciclos comuns não pode ser rejeitada, a um nível de significância de $5 \%$, e consequentemente é confirmada a presença de um vetor de cocaracterização na amostra.

Tabela 7: Teste de ciclos comuns

\begin{tabular}{lccc}
\hline No de Ciclos $^{\circ}$ & Estatística LR & Graus de Liberdade & p-valor \\
\hline 7,0000 & 8,1792 & 8,0000 & 0,4162 \\
\hline Fonte: Elaborado pelos autores. A ordem de defasagem \\
selecionada para estimação do VEC e por consequência, do teste \\
de ciclos comuns, segue o critério de Akaike. A escolha desse \\
critério se deu porque o teste só pode ser realizado em um VEC \\
de ordem igual ou superior a dois.
\end{tabular}

A existência de um vetor de cocaracterização e sete vetores de cointegração entre as séries implica que a matriz $A=\left[\tilde{\alpha}^{\prime} \alpha^{\prime}\right]$ possui posto pleno, logo é possível obter as tendências e ciclos individuais por meio da partição da matriz $A$, assim como encontrar os ciclos comuns pela combinação dos vetores de cointegração e das variáveis do sistema $\left(\alpha^{\prime} Y_{t}=\alpha^{\prime} C_{t}\right)$.

\subsection{Tendências Comuns}

De acordo com o que foi discutido anteriormente, o fato da matriz $A=\left[\tilde{\alpha}^{\prime} \alpha^{\prime}\right]$ possuir posto pleno nos permite utilizar a condição especial verificada na Equação (10). A partir daí pode-se estimar as tendências individuais, $\tilde{\alpha}-$ $\left(\tilde{\alpha}^{\prime} Y_{t}\right)$, bem como a $n-r=8-7=1$ tendência comum, $\tilde{\alpha}^{\prime} Y_{t}$.

A tendência comum \#1 corrobora a hipótese de estabilidade na evolução temporal do índice de inflação para o período pós 2004, com a taxa de inflação oscilando em torno de uma média. A série apresentou picos negativos e positivos na taxa de variação acumulada anual de inflação no segundo semestre de 1998 e 2002, respectivamente.

Observando a Figura 3 observa-se que todas as tendências individuais apresentam trajetórias semelhantes, havendo um comportamento médio, com as regiões metropolitanas do Nordeste apresentando patamares superiores durante o período da crise de confiança, enquanto as regiões metropolitanas do sudeste destacam-se superiormente no período de relativa estabilização pós 2004. Cabe destacar também, que as tendências individuais apresentaram um menor nível de taxa de inflação no período final de 2006, época também observada pela tendência comum.

A trajetória semelhante entre os movimentos das tendências individuais é corroborada pelos elevados índices de correlação positiva entre as regiões me- 
Tendências e Ciclos Comuns entre as Inflações das Regiões Metropolitanas do Brasil 385

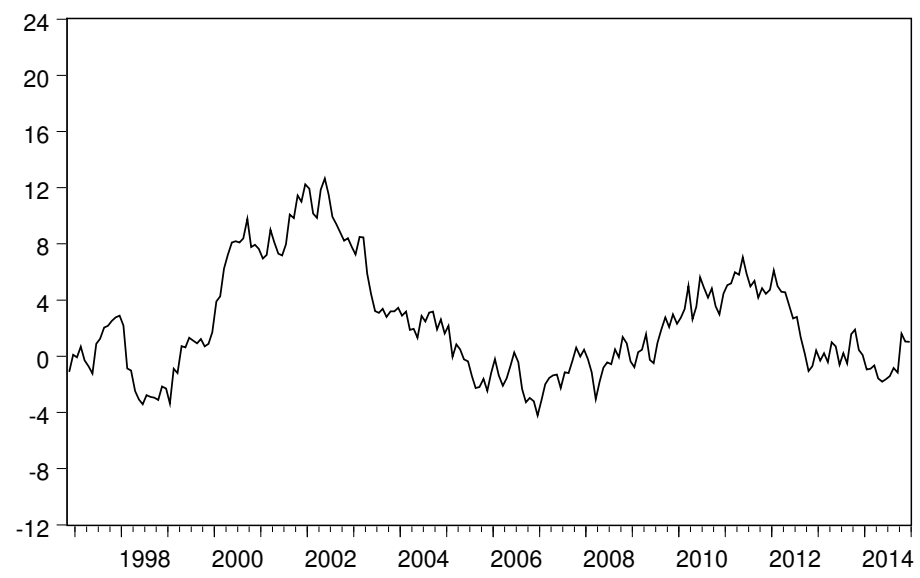

Fonte: Elaborado pelos autores.

Figura 2: Tendência comum

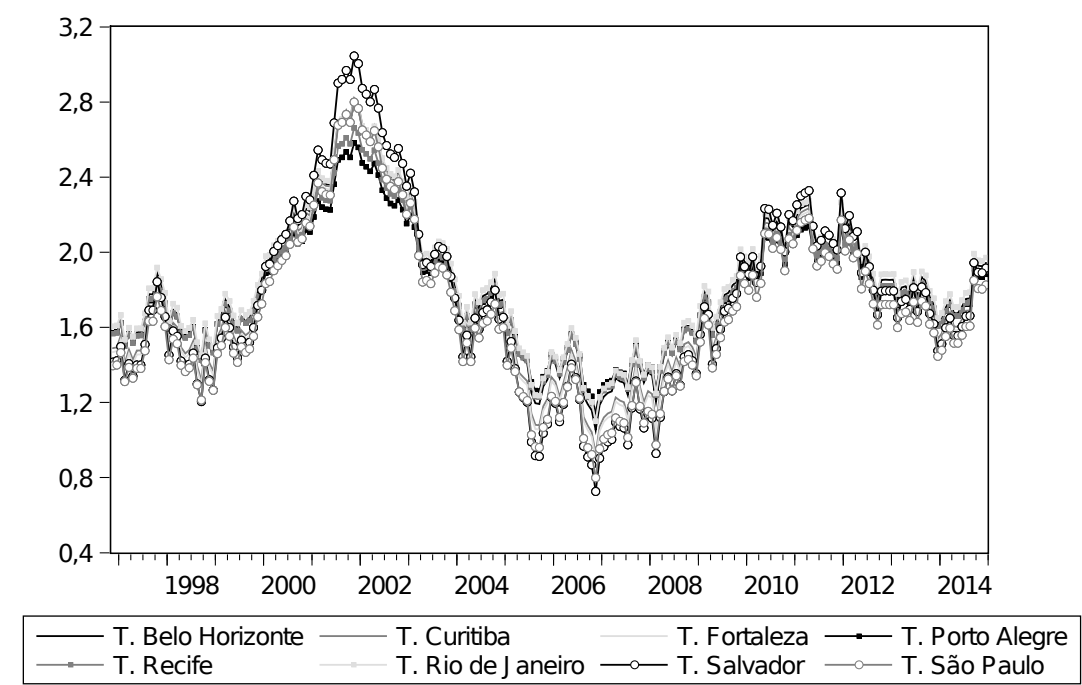

Fonte: Elaborado pelos autores.

Figura 3: Tendências individuais 
tropolitanas - todos os índices de correlação são superiores a 0,99. A tendência comum também apresenta nível de correlação positiva com as tendências individuais, observando-se um nível médio de correlação superior à 0,90.

\subsection{Ciclos Comuns}

Feita a análise de comovimentos de longo prazo, a próxima etapa do presente trabalho será extrair os sete ciclos comuns $(n-s=8-1=7)$ a partir de $\alpha^{\prime} Y_{t}=\alpha^{\prime} C_{t}$, e os componentes de ciclos individuais de cada região metropolitana por meio da equação $\alpha^{-}\left(\alpha^{\prime} Y_{t}\right)$ nas Figuras 4, 5 e A.1. Cabe fazer uma ressalva que, devido à delimitação de espaço, somente os ciclos comuns \#1 e \#7 serão representados aqui pelo fato de ambos terem sido os únicos a possuírem valores relevantes de correlação com os ciclos individuais.

Assim como na análise das tendências das regiões metropolitanas, os ciclos individuais possuem um padrão temporal similar. Já os ciclos comuns apresentaram uma dinâmica temporal menos volátil do que os ciclos individuais, com destaque para o ciclo comum \#1, o qual apresentou desvio padrão de 0,190 . Analisando os valores extremos destaca-se a região metropolitana de São Paulo, com o segundo maior valor máximo da amostra $(1,148)$ e o valor mínimo mais acentuado $(-1,618)$ bem como o maior nível de volatilidade $(0,504)$.

É possível visualizar maiores detalhes desses componentes cíclicos ao utilizar a análise de correlação cruzada de forma a avaliar a relação mútua entre as variáveis.

De acordo com a Tabela 9 o ciclo comum \#1 se relaciona de forma negativa com os ciclos de Curitiba e São Paulo, sendo correlacionado de forma positiva com os demais ciclos individuais. Em termos de magnitude, o ciclo comum \#1 apresentou níveis mais elevados de correlação com os ciclos da região Nordeste e de Belo Horizonte. Já o ciclo comum \#7 apresentou correlação negativa com os ciclos individuais, indicando que os movimentos transitórios causados pelo ciclo comum \#7 nos ciclos individuais ocorrem na mesma direção, diferindo apenas em intensidade (elevadamente correlacionado com os ciclos da região Sul e São Paulo). Já a correlação entre os ciclos individuais oscila entre 0,705 e 0,912 .

Devido às altas correlações entre os ciclos individuais, e os níveis moderados de correlação entre os ciclos comuns e ciclos individuais, o teste de causalidade de Granger se torna uma ferramenta interessante para verificar a capacidade preditiva dos ciclos individuais entre si e a importância dos ciclos comuns \#1 e \#7 na previsão dos ciclos individuais.

A análise do teste de causalidade entre os ciclos individuais sugere a presença de precedência temporal do ciclo de São Paulo em direção aos ciclos de Belo Horizonte, Curitiba e Recife, havendo também uma relação de causalidade bidirecional entre o ciclo paulista e o ciclo do Rio de Janeiro. Os ciclos de Recife e Salvador não apresentaram capacidade preditiva sobre nenhum dos demais ciclos individuais. Destaca-se também que, assim como o ciclo de Fortaleza, o ciclo de Salvador não foi previsto por nenhum dos ciclos individuais.

Conforme a Tabela 11, nenhum dos ciclos individuais foi capaz de prever o ciclo comum \#7, enquanto o ciclo comum \#7 somente foi capaz de prever o ciclo do Rio de Janeiro. Já em relação ao ciclo comum \#1, observa-se que o ciclo de São Paulo foi o único capaz de o prever. 
Figura 4: Ciclos comuns e individuais

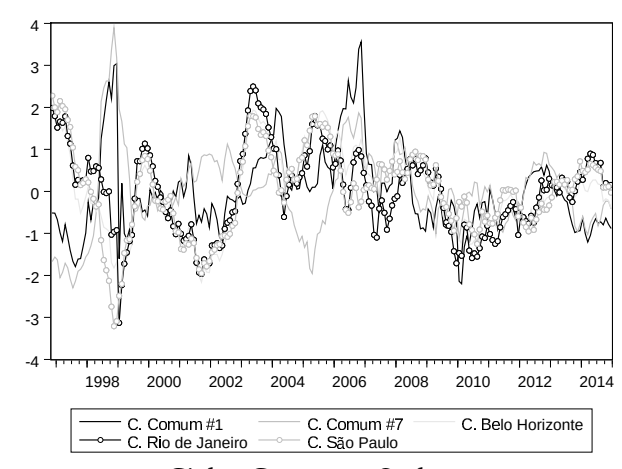

Ciclos Comuns e Sudeste

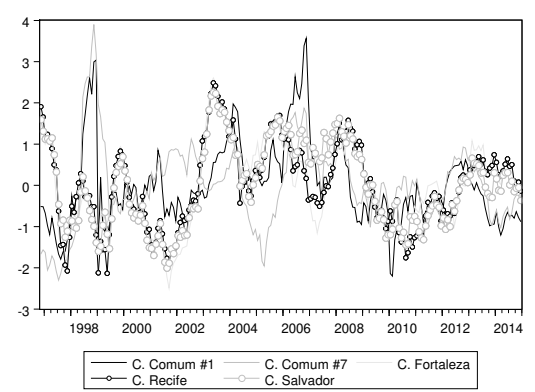

Ciclos Comuns e Nordeste

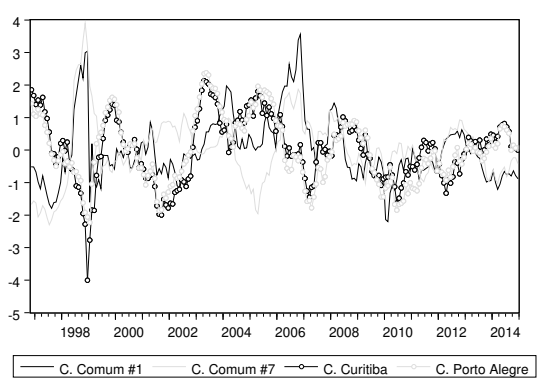

Ciclos Comuns e Sul

Fonte: Elaborado pelos autores. 
Tabela 8: Estatísticas descritivas dos ciclos

\begin{tabular}{lcccccccccc}
\hline Métrica & Comum \#1 & Comum \#7 & BH & CUR & \multicolumn{1}{c}{ FOR } & POA & REC & \multicolumn{1}{c}{ RJ } & \multicolumn{1}{c}{ SAL } & SP \\
\hline Média & 0,358 & $-0,155$ & 0,000 & 0,000 & 0,000 & 0,000 & 0,000 & 0,000 & 0,000 & 0,000 \\
Máximo & 1,035 & 1,216 & 0,900 & 0,863 & 1,098 & 0,955 & 1,063 & 0,916 & 1,024 & 1,148 \\
Mínimo & $-0,061$ & $-0,962$ & $-1,394$ & $-1,620$ & $-1,052$ & $-0,895$ & $-0,914$ & $-1,150$ & $-0,908$ & $-1,618$ \\
Desvio Padrão & 0,190 & 0,352 & 0,460 & 0,404 & 0,422 & 0,401 & 0,428 & 0,367 & 0,453 & 0,504 \\
\hline
\end{tabular}

Fonte: Elaborado pelos autores. 
Tabela 9: Correlação entre os ciclos individuais e comuns

\begin{tabular}{|c|c|c|c|c|c|c|c|c|c|c|}
\hline Ciclos & Comum \#1 & Comum \#7 & BH & CUR & FOR & POA & REC & RJ & SAL & SP \\
\hline Comum \#1 & 1,000 & & & & & & & & & \\
\hline Comum \#7 & 0,677 & 1,000 & & & & & & & & \\
\hline $\mathrm{BH}$ & 0,317 & $-0,313$ & 1,000 & & & & & & & \\
\hline CUR & $-0,007$ & $-0,555$ & 0,866 & 1,000 & & & & & & \\
\hline FOR & 0,288 & $-0,149$ & 0,866 & 0,825 & 1,000 & & & & & \\
\hline POA & 0,045 & $-0,422$ & 0,795 & 0,918 & 0,836 & 1,000 & & & & \\
\hline REC & 0,333 & $-0,078$ & 0,874 & 0,776 & 0,940 & 0,793 & 1,000 & & & \\
\hline RJ & 0,211 & $-0,344$ & 0,892 & 0,863 & 0,830 & 0,870 & 0,857 & 1,000 & & \\
\hline SAL & 0,408 & $-0,007$ & 0,919 & 0,725 & 0,881 & 0,705 & 0,923 & 0,807 & 1,000 & \\
\hline SP & $-0,101$ & $-0,623$ & 0,911 & 0,912 & 0,782 & 0,815 & 0,772 & 0,844 & 0,787 & 1,000 \\
\hline
\end{tabular}

Fonte: Elaborado pelos autores. 
Tabela 10: Causalidade de Granger entre os ciclos individuais (b)

\begin{tabular}{lcccccccc}
\hline Causalidade & BH & CUR & FOR & POA & REC & RJ & SAL & SP \\
\hline BH & & 0,957 & 0,344 & $0,014^{*}$ & 0,493 & 0,097 & 0,408 & 0,361 \\
CUR & $0,002^{*}$ & & 0,427 & 0,431 & $0,012^{*}$ & $0,000^{*}$ & 0,457 & 0,871 \\
FOR & 0,109 & 0,196 & & 0,818 & $0,034^{*}$ & $0,017^{*}$ & 0,178 & 0,366 \\
POA & $0,024^{*}$ & $0,002^{*}$ & 0,388 & & $0,013^{*}$ & $0,000^{*}$ & 0,267 & 0,435 \\
REC & 0,938 & 0,602 & 0,752 & 0,097 & & 0,124 & 0,195 & 0,229 \\
RJ & 0,117 & 0,096 & 0,108 & $0,000^{*}$ & 0,810 & & 0,930 & $0,005^{*}$ \\
SAL & 0,311 & 0,719 & 0,815 & 0,219 & 0,418 & 0,185 & & 0,430 \\
SP & $0,005^{*}$ & $0,010^{*}$ & 0,897 & 0,614 & $0,078^{*}$ & $0,000^{*}$ & 0,165 & \\
\hline
\end{tabular}

Fonte: Elaborado pelos autores. Teste de causalidade com uma defasagem. (b) Tabela reporta o p-valor do teste. * Significância de 5\%.

Tabela 11: Causalidade de Granger entre os ciclos individuais e comuns (b)

\begin{tabular}{ccccccccc}
\hline Causalidade & BH & CUR & FOR & POA & REC & RJ & SAL & SP \\
\hline C. Comum\#1 > C. Individual & $0,005^{*}$ & $0,012^{*}$ & 0,163 & $0,003^{*}$ & 0,059 & $0,001^{*}$ & 0,194 & 0,361 \\
C. Individual > C. Comum\#1 & 0,258 & 0,070 & 0,300 & 0,199 & 0,790 & 0,222 & 0,257 & $0,029^{*}$ \\
C. Comum\#7 > C. Individual & 0,174 & 0,190 & 0,961 & 0,509 & 0,164 & $0,009^{*}$ & 0,170 & 0,445 \\
C. Individual > C. Comum\#7 & 0,607 & 0,985 & 0,833 & 0,922 & 0,174 & 0,122 & 0,620 & 0,624 \\
\hline
\end{tabular}

Fonte: Elaborado pelos autores. Teste de causalidade com uma defasagem. (b) Tabela reporta o p-valor do teste. *Significância de $5 \%$.

Tal resultado se alinha a hipótese de contágio do ciclo de São Paulo em direção aos ciclos de Belo Horizonte e Curitiba, visto que o ciclo comum \#7 apresentou capacidade preditiva sobre os mesmos.

\subsection{Restrição Teórica e Teste de Cointegração}

Conforme foi observado na Seção 4.3, as séries de inflação das regiões metropolitanas do Brasil apresentaram uma tendência estocástica comum, com a matriz de cointegração normalizada sendo representada da seguinte forma:

$$
\alpha=\left[\begin{array}{ccccccc}
1 & 0 & 0 & 0 & 0 & 0 & 0 \\
0 & 1 & 0 & 0 & 0 & 0 & 0 \\
0 & 0 & 1 & 0 & 0 & 0 & 0 \\
0 & 0 & 0 & 1 & 0 & 0 & 0 \\
0 & 0 & 0 & 0 & 1 & 0 & 0 \\
0 & 0 & 0 & 0 & 0 & 1 & 0 \\
0 & 0 & 0 & 0 & 0 & 0 & 1 \\
-0,869 & -0,947 & -0,952 & -0,718 & -0,779 & -0,850 & -1,159
\end{array}\right]
$$

Visto que todas as elasticidades de longo prazo estimadas possuem valores próximas da unidade em termos absolutos, uma representação usual do vetor de cointegração normalizado, na presença de uma única tendência estocástica comum é a imposição de restrições sobre os parâmetros do espaço de cointegração, construindo um vetor estrutural de modo que os choques permanentes exerçam impactos equivalentes em todas as regiões ${ }^{5}$. Este subespaço de

\footnotetext{
tigo.

${ }^{5}$ Agradecemos ao parecerista pela sugestão da restrição teórica implantada no presente ar-
} 
cointegração é identificado teoricamente como:

$$
\alpha=\left[\begin{array}{ccccccc}
1 & 0 & 0 & 0 & 0 & 0 & 0 \\
0 & 1 & 0 & 0 & 0 & 0 & 0 \\
0 & 0 & 1 & 0 & 0 & 0 & 0 \\
0 & 0 & 0 & 1 & 0 & 0 & 0 \\
0 & 0 & 0 & 0 & 1 & 0 & 0 \\
0 & 0 & 0 & 0 & 0 & 1 & 0 \\
0 & 0 & 0 & 0 & 0 & 0 & 1 \\
-1 & -1 & -1 & -1 & -1 & -1 & -1
\end{array}\right]
$$

Para verificar a validade do vetor de cointegração restrito, empregou-se o teste de restrição de Wald usando o estimador de verossimilhança proposto em Johansen et al. (2000) robusto à presença de quebra estrutural no sistema. $O$ resultado do teste $(\mathrm{p}$-valor $=0,000)$ rejeitou a hipótese nula de que o vetor estrutural constitui uma base para o espaço de cointegração, indicando que as elasticidades de longo prazo são estatisticamente diferentes da unidade.

Apesar da rejeição do ponto de vista estatístico, implementou-se novamente o exercício empírico a partir do vetor de cointegração restrito.

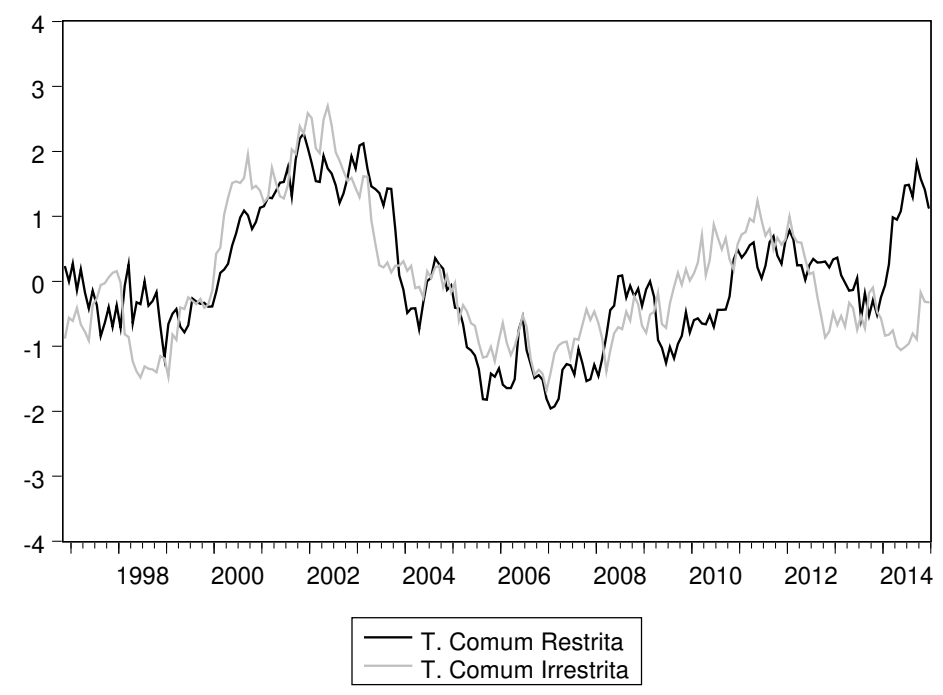

Fonte: Elaborado pelos autores.

Figura 5: Tendência comum restrita e irrestrita

A Figura 5 demonstra que as tendências comums estimadas possuem um comportamento temporal próximo até o último período de 2013, a partir daí a tendência comum do modelo restrito indica uma trajetória de crescimento na taxa de inflação, enquanto a tendência comum empírica indica um crescimento na taxa de inflação menos acentuado, ocorrendo somente a partir do segundo semestre de 2014. A correlação entre as tendências comuns restrito e irrestrito foi de 0,71 , indicando relação linear robusta entre os componentes.

Com relação a dinâmica de curto prazo, a segunda análise empírica corrobora em grande parte as evidências reportadas anteriormente. 
1. Os ciclos individuais novamente apresentaram-se fortemente correlacionados, com os valores oscilando entre 0,682 (CUR-SAL) e 0,912 (FORREC). No entanto, os coeficientes de correlação indicam a existência de uma maior interação entre os ciclos da região Sul e Sudeste (correlações superiores à 0,82 ), enquanto as unidades da região Nordeste apresentaram correlações inferiores à $0,80 \mathrm{com}$ as regiões metropolitanas do Sul e Sudeste (a exceção da relação FOR-POA, na qual a correlação foi igual à $0,82)$.

2. Somente o ciclo comum \#1 apresentou níveis relevantes de correlação com os ciclos individuais. Observou-se a presença de correlação positiva entre o ciclo comum \#1 todos os ciclos individuais, com o mesmo possuindo níveis mais expressivos de correlação com os ciclos da região Sul e Sudeste $(0,43$ e 0,46 em média, respectivamente).

3. Os ciclos individuais de Curitiba e Belo Horizonte se mostraram ambos previsíveis pelo ciclo de São Paulo, enquanto o ciclo de São Paulo não foi previsto pelos ciclos de Curitiba e Belo Horizonte. A exceção da capacidade de previsão do ciclo de Fortaleza em direção ao ciclo de Recife, todas as demais relações de causalidade relatadas na Seção 4.6 foram confirmadas via modelo restrito. Já o ciclo comum \#1 não apresentou capacidade preditiva sobre nenhum dos ciclos individuais, bem como não foi previsto pelos mesmos.

\section{Considerações Finais}

O objetivo deste artigo foi caracterizar os relacionamentos de curto e de longo prazo entre as taxas de inflação das capitais, incluindo as regiões metropolitanas, da Bahia, Ceará, Minas Gerais, Pernambuco, Paraná, Rio de Janeiro, Rio Grande do Sul e São Paulo no período de outubro de 1995 a dezembro de 2014; ou seja, avaliar se as principais regiões metropolitanas do Brasil compartilham de tendências e ciclos comuns em termos de dinâmica inflacionária.

A análise mostrou que, mesmo na presença de uma quebra estrutural em outubro de 2003, as séries temporais das regiões metropolitanas são cointegradas, compartilhando de uma relação de equilíbrio de longo prazo. Essa evidência corrobora a hipótese de existência de uma única tendência comum entre as inflações regionais. As elasticidades de longo prazo estimadas indicaram que as regiões respondem a choques permanentes em igual direção.

Assim, em termos de estratégia e condução de política monetária, o fato das regiões compartilharem uma única tendência comum ns sugere que as políticas monetárias implantadas exercem efeitos permanentes similares nas taxas de inflação de todas as regiões metropolitanas estudadas.

Em termos de dinâmica de curto prazo, viu-se que as regiões da amostra compartilham de sete ciclos comuns. A análise de correlação apontou, ainda, que existe uma associação linear positiva entre os ciclos das diferentes regiões metropolitanas do Brasil.

O teste de causalidade apurou que o ciclo de São Paulo causa, no sentido de Granger, os ciclos de Curitiba e Belo Horizonte a um nível de significância de $5 \%$, indicando a possibilidade de contágio da dinâmica de curto prazo do ciclo paulistano em direção aos ciclos de Curitiba e Belo Horizonte. Verificouse também que nenhum dos ciclos individuais foi capaz de prever o ciclo co- 
mum \#1, bem como o ciclo comum \#1 não foi capaz de prever nenhum dos ciclos individuais. Já o ciclo individual de São Paulo possui uma relação de precedência temporal em relação a dinâmica do ciclo comum \#7.

Dessa forma, a capacidade preditiva do ciclo de São Paulo em relação ao ciclo comum \#7 corrobora a hipótese de contágio do mesmo em direção aos ciclos de Curitiba e Belo Horizonte, uma vez que o ciclo comum \#7 apresenta precedência temporal em relação aos ciclos das regiões metropolitanas de $\mathrm{Cu}$ ritiba e Belo Horizonte.

O segundo exercício empírico, realizado por meio de uma restrição teórica sobre o vetor de cointegração, confirmou a existência de contágio do ciclo individual paulistano sobre os ciclos de Curitiba e Belo Horizonte. A análise indicou também forte correlação entre os ciclos individuais, evidenciando uma maior interação na dinâmica de curto prazo dos ciclos da região Sul e Sudeste.

As fortes semelhanças nos ciclos das taxas de inflação de diferentes regiões metropolitanas por um lado tendem a fortalecer o efeito de choques nacionais e regionais por meio da propagação destes entre as regiões, por outro lado essa dinâmica semelhante no curto prazo pode auxiliar o balizamento de estratégias de políticas monetárias que tragam resultados efetivos para a estabilização inflacionária em todas as regiões do país estudadas. 


\section{Referências Bibliográficas}

Athanasopoulos, G., De Carvalho Guillén, O. T., Issler, J. V. \& Vahid, F. (2011), 'Model selection, estimation and forecasting in VAR models with short-run and long-run restrictions', Journal of Econometrics 164(1), 116-129. URL: $h t t p: / / d x$.doi.org/10.1016/j.jeconom.2011.02.009

Banerjee, A., Lumsdaine, R. L. \& Stock, J. H. (1992), 'Recursive and sequential tests of the unit-root and trend-break hypotheses: theory and international evidence', Journal of Business and Economic Statistics 10(3), 271-287.

Baxter, M. \& King, R. G. (1999), 'Measuring business cycles: approximate band-pass filters for economic time series', Review of Economics and Statistics 81(4), 575-593.

URL: http://www.mitpressjournals.org/doi/10.1162/003465399558454

Beck, G. W., Hubrich, K., Marcellino, M. \& Adam, K. (2009), 'Centre for economic policy research center for economic studies maison des sciences de l'homme', Economic Policy 24(57), 141-184.

Beveridge, S. \& Nelson, C. R. (1981), 'A new approach to decomposition of economic time series into permanent and transitory components with particular attention to measurement of the 'business cycle", Journal of Monetary Economics 7(2), 151-174.

Carlino, G. \& Sill, K. (2001), 'Regional income fluctuations: common trends and common cycles', Review of Economics and Statistics 83(3), 446-456.

URL: http://www.mitpressjournals.org/doi/10.1162/00346530152480090

Cerro, A. M. \& Pineda, J. (2002), 'Do common cycles exist in Latin American Countries?', Latin American Research Abstracts pp. 813-836.

Elliott, G., Rothenberg, T. J. \& Stock, J. H. (1992), Efficient Tests for an Autoregressive Unit Root.

Gali, J. \& Gertler, M. (1999), 'Inflation dynamics: a structural econometric analysis', Journal of Monetary Economics 44(2), 195-222.

Gerard, H. (2012), 'Discussion Paper Co-movement in Inflation'.

Giles, D. E. \& Godwin, R. T. (2012), 'Testing for multivariate cointegration in the presence of structural breaks: p-values and critical values', Applied Economics Letters 19(16), 1561-1565.

Gonzalo, J. \& Granger, C. (1995), 'Estimation of common long-memory components in cointegrated systems', Journal of Business and Economic Statistics 13(1), 27-35.

Gutierrez, C. E. C. \& Gomes, F. A. R. (2009), 'Evidence on common feature and business cycle synchronization in', Brazilian Review of Econometrics 29(1), 37-58.

Hairault, J.-O. \& Portier, F. (1993), 'Money, New Keynesian macroeconomics, and the business cycle', European Economic Review 37, 33-68. 
Hecq, A. (2002), 'Common trends and common cycles in Latin America', Medium Econometrische Toepassingen 10(3), 12.

URL: resolver.scholarsportal.info/resolve/08837252/v08i0004/341_ctacc

Hodrick, R. J. \& Prescott, E. C. (1981), Post-War U.S. Business Cycles: An Empirical Investigation.

Issler, J. V. \& Vahid, F. (2001), 'Common cycles and the importance of transitory shocks to macroeconomic aggregates', Journal of Monetary Economics 47(3), 449-475.

Johansen, S. (1988), 'Statistical analysis of cointegration vectors', Journal of Economic Dynamics and Control 12(2-3), 231-254.

Johansen, S., Mosconi, R. \& Nielsen, B. (2000), 'Cointegration analysis in the presence of structural breaks in the deterministic trend', Econometrics Journal 3(2), pp. 216-49.

Mankiw, N. G. (1989), 'Real business cycles: a New Keynesian perspective', American Economic Association 3(3), 79-90.

Manopimoke, P. (2012), Hong Kong Inflation Dynamics: Trend and Cycle Relationships with the U.S. and China.

Mills, T. C. \& Holmes, M. J. (1999), 'Common trends and cycles in European industrial production: exchange rate regimes and economic convergence', Manchester School 67(4), 557-587.

Perron, B. Y. P. (1989), 'The great crash, the oil price shock, and the unit root hypothesis', The Econometric Society 57(6), 1361-1401.

Perron, P. \& Vogelsang, T. J. (1992), 'Nonstationarity and the level shift with an application to purchasing power parity', Journal of Business and Economic Statistics 10(3), 301-320.

Roache, S. (2008), 'Central America's regional trends and US cyckes', Economic Progress and Reforms pp. 34-60.

Shaoping, W. \& Xiaotao, S. (2014), 'Codependent cycles of Chinese inflation', Social Sciences in China 35(4), 31-45.

Vahid, F. \& Engle, R. F. (1993), 'Common trends and common cycles', Journal of Applied Econometrics 8(4), 341-360.

Vogelsang, T. J. \& Perron, P. (1998), 'Additional tests for a unit root allowing for a break in the trend function at an unknown time', International Economic Review 39(4), 1073-1100.

Warne, A. (2008), Estimation and Testing for Common Cycles.

Zivot, E. \& Andrews, D. W. (1992), 'Further evidence on the great crash, the oil price shock, and the unit root hypothesis', Journal of Business E Economic Statistics 10(3), 251-270.

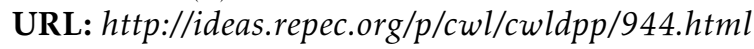

\section{Apêndice $A$}


Figura A.1: Função autocorrelação (FAC) e função autocorrelação parcial dos resíduos $(\rho=1)$

Resíduo BH

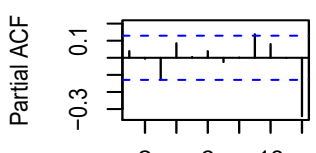

Lag

Resíduo BH

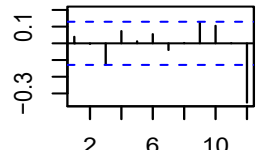

Resíduo REC

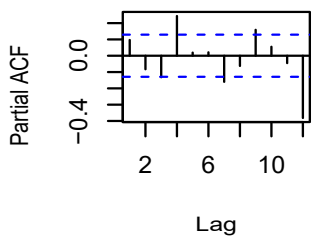

Resíduo REC

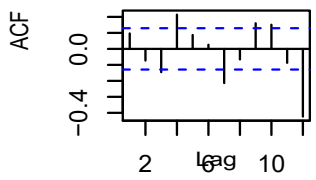

Resíduo CUR

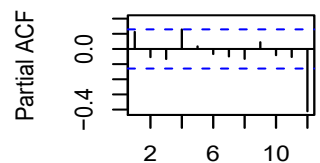

Lag
Resíduo FOR

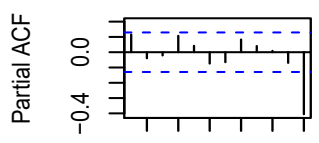

Lag

Resíduo POA

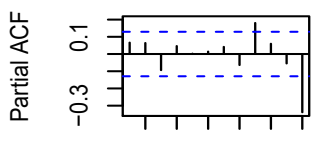

Lag

\section{Resíduo POA}

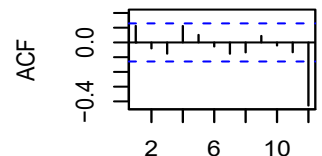

Lag

Resíduo RJ

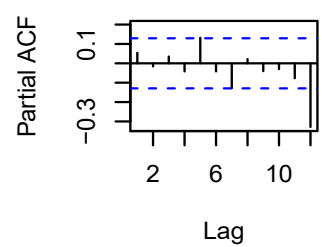

Resíduo RJ

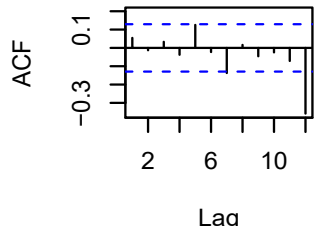

Lag

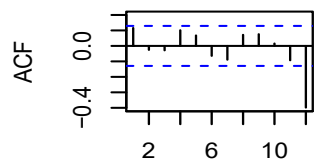

Lag

Resíduo SAL

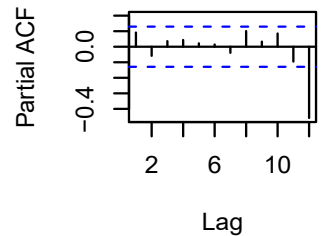

Resíduo SAL

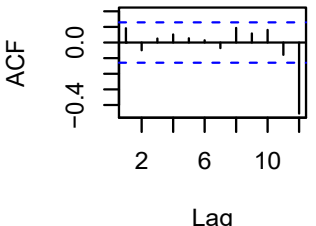

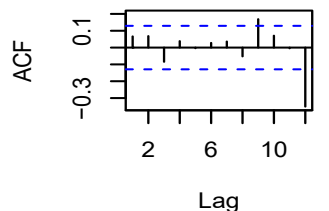

Resíduo SP

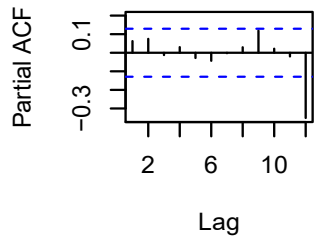

Resíduo SP

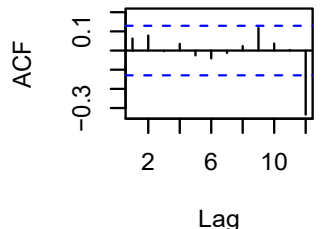


Figura A.2: Teste de correlação serial sobre os resíduos $(\rho=1)$

Resíduo BH

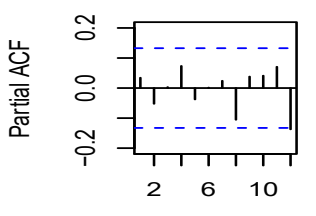

Lag
Resíduo CUR

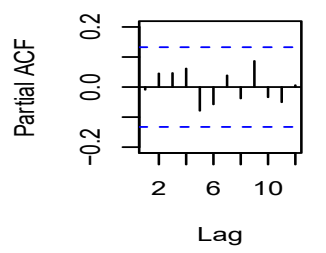

Resíduo CUR

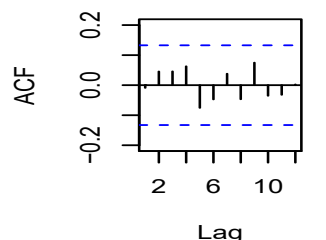

Resíduo RJ

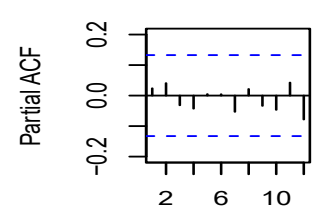

Lag

\section{Resíduo REC}

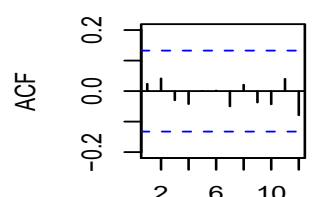

Lag

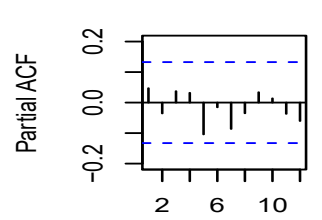

Lag

\section{Resíduo RJ}

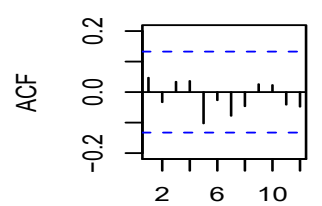

Lag

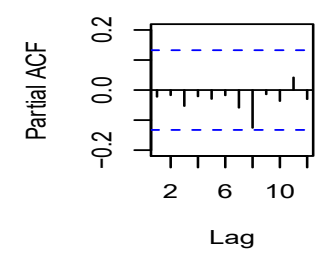

Resíduo FOR

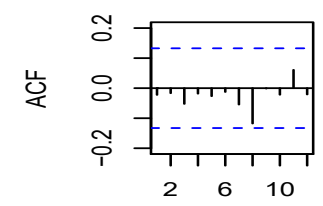

Lag

Resíduo SAL

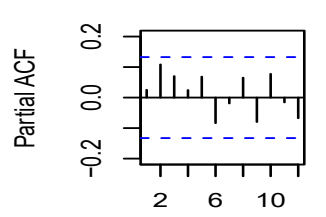

Lag

Resíduo SAL

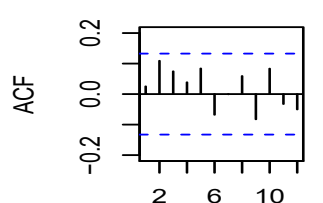

Lag

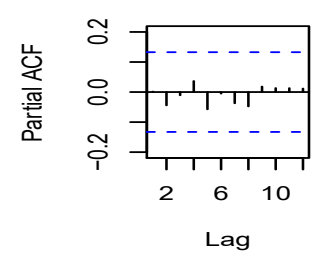

Resíduo POA

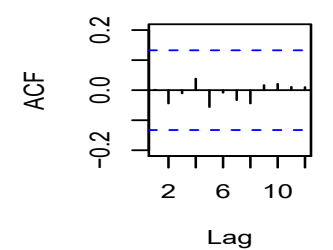

Resíduo SP

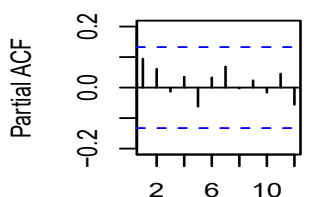

Lag

\section{Resíduo SP}

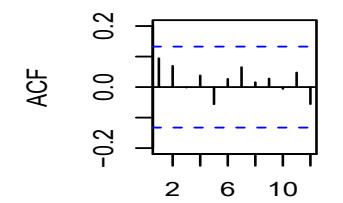

Lag

Fonte: Elaborado pelos autores. * Rejeição da hipótese nula a 5\%. Estatística $\chi^{2}$ reportada com o respectivo p-valor entre parênteses. 
Tabela A.1: Critério de seleção de defasagens

\begin{tabular}{lccrrr}
\hline Defasagens & LR & FPE & AIC & \multicolumn{1}{c}{ SC } & \multicolumn{1}{c}{ HQ } \\
\hline 0 & NA & 3,267436 & 23,887020 & 24,009640 & 23,936530 \\
1 & 3423,682000 & $6,08 \mathrm{e}-07$ & 8,389973 & $9,493544^{*}$ & 8,835527 \\
2 & 204,684600 & $4,00 \mathrm{e}-07$ & 7,968088 & 10,052610 & $8,809689^{*}$ \\
3 & 96,496000 & $4,38 \mathrm{e}-07$ & 8,054837 & 11,120310 & 9,292485 \\
4 & 107,409700 & $4,46 \mathrm{e}-07$ & 8,063109 & 12,109540 & 9,696804 \\
5 & 114,996900 & $4,28 \mathrm{e}-07$ & 8,004344 & 13,031720 & 10,034090 \\
6 & 97,255840 & $4,45 \mathrm{e}-07$ & 8,018748 & 14,027080 & 10,444540 \\
7 & 79,074120 & $5,08 \mathrm{e}-07$ & 8,116087 & 15,105370 & 10,937920 \\
8 & 88,695970 & $5,39 \mathrm{e}-07$ & 8,127721 & 16,097960 & 11,345610 \\
9 & 113,765400 & $4,75 \mathrm{e}-07$ & 7,940772 & 16,891960 & 11,554700 \\
10 & 122,658000 & $3,84 \mathrm{e}-07$ & 7,647434 & 17,579570 & 11,657410 \\
11 & 142,501300 & $2,58 \mathrm{e}-07$ & 7,152572 & 18,065660 & 11,558600 \\
12 & $107,676900^{*}$ & $2,20 \mathrm{e}-07^{*}$ & $6,867734^{*}$ & 18,761780 & 11,669810 \\
\hline
\end{tabular}

Fonte: Elaborado pelos autores. ${ }^{*}$ Indica a ordem de defasagem selecionada pelo critério de informação. 
Tabela A.2: Teste de correlação serial sobre os resíduos $(\rho=1)$

\begin{tabular}{|c|c|c|c|c|c|c|c|c|}
\hline & BH & CUR & FOR & POA & REC & RJ & SAL & SP \\
\hline $\begin{array}{l}\text { Teste de Breusch-Godfrey. } \\
\text { (H0: Não há correlação serial) }\end{array}$ & $\begin{array}{c}38,91^{*} \\
(4,54 e-03)\end{array}$ & $\begin{array}{c}62,21^{*} \\
(1,72 e-06)\end{array}$ & $\begin{array}{c}58,05^{*} \\
(7,83 e-06)\end{array}$ & $\begin{array}{c}42,04^{*} \\
(1,74 e-03)\end{array}$ & $\begin{array}{c}66,19^{*} \\
(3,91 e-07)\end{array}$ & $\begin{array}{c}39,32^{*} \\
(4,00 e-03)\end{array}$ & $\begin{array}{c}57,89^{*} \\
(8,30 e-06)\end{array}$ & $\begin{array}{c}46,06^{*} \\
(4,86 e-04)\end{array}$ \\
\hline
\end{tabular}
$5 \%$ 
Tabela A.3: Teste de correlação serial sobre os resíduos $(\rho=12)$

\begin{tabular}{lcccccccr}
\hline & BH & CUR & FOR & POA & REC & RJ & SAL & SP \\
\hline Teste de Breusch-Godfrey & & & & & & & & \\
(H0: Não há correlação serial) & 12,74 & 8,81 & 7,28 & 4,77 & 7,52 & 9,56 & 16,91 & 8,29 \\
& $(0,851)$ & $(0,976)$ & $(0,992)$ & $(0,999)$ & $(0,991)$ & $(0,963)$ & $(0,596)$ & $(0,984)$ \\
\hline
\end{tabular}

Fonte: Elaborado pelos autores. Estatística $\chi^{2}$ reportada com o respectivo p-valor entre parênteses. * Rejeição da hipótese nula a 5\%.

Tabela A.4: Estatística descritiva dos ciclos (modelo restrito)

\begin{tabular}{|c|c|c|c|c|c|c|c|c|c|c|}
\hline Métrica & Comum \#1C & Comum \#7 & BH & CUR & FOR & POA & REC & RJ & SAL & SP \\
\hline Média & 0,144 & 0,097 & 0,000 & 0,000 & 0,000 & 0,000 & 0,000 & 0,000 & 0,000 & 0,000 \\
\hline Máximo & 0,974 & 0,795 & 1,076 & 0,782 & 0,842 & 1,022 & 0,942 & 0,755 & 0,842 & 0,929 \\
\hline Mínimo & $-0,283$ & $-0,512$ & $-1,292-$ & $-1,482-$ & $-0,907-$ & $-0,830-$ & $-0,877$ & $-1,044$ & $-0,784-$ & $-1,676$ \\
\hline Desvio Padrão & 0,219 & 0,188 & 0,455 & 0,364 & 0,378 & 0,386 & 0,407 & 0,334 & 0,371 & 0,470 \\
\hline
\end{tabular}

Fonte: Elaborado pelos autores. 
Tabela A.5: Correlação entre os ciclos individuais e comuns (modelo restrito)

\begin{tabular}{lcccccccccc}
\hline Ciclos & Comum \#1 & Comum \#7 & BH & CUR & FOR & POA & REC & RJ & SAL & SP \\
\hline Comum \#1 & 1,000 & & & & & & & & & \\
Comum \#7 & 0,240 & 1,000 & & & & & & & & \\
BH & 0,458 & $-0,189$ & 1,000 & & & & & & & \\
CUR & 0,408 & $-0,028$ & 0,835 & 1,000 & & & & & & \\
FOR & 0,262 & $-0,203$ & 0,842 & 0,784 & 1,000 & & & & & \\
POA & 0,452 & 0,006 & 0,836 & 0,892 & 0,832 & 1,000 & & & & \\
REC & 0,247 & $-0,226$ & 0,881 & 0,696 & 0,913 & 0,786 & 1,000 & & & \\
RJ & 0,470 & 0,015 & 0,906 & 0,814 & 0,790 & 0,870 & 0,831 & 1,000 & & \\
SAL & 0,304 & $-0,265$ & 0,879 & 0,683 & 0,858 & 0,711 & 0,901 & 0,763 & 1,000 & \\
SP & 0,446 & $-0,028$ & 0,881 & 0,909 & 0,747 & 0,820 & 0,729 & 0,827 & 0,748 & 1,000 \\
\hline
\end{tabular}

Fonte: Elaborado pelos autores. 
Tabela A.6: Causalidade de Granger entre os ciclos individuais (modelo restrito) (b)

\begin{tabular}{llllllllc}
\hline Causalidade & BH & CUR & FOR & POA & REC & RJ & SAL & SP \\
\hline BH & & 0,945 & 0,460 & 0,052 & 0,265 & $0,020^{*}$ & 0,341 & 0,442 \\
CUR & $0,010^{*}$ & & 0,578 & 0,569 & $0,026^{*}$ & $0,000^{*}$ & 0,421 & 0,945 \\
FOR & 0,189 & 0,186 & & 0,961 & 0,051 & $0,042^{*}$ & 0,141 & 0,460 \\
POA & $0,038^{*}$ & $0,004^{*}$ & 0,554 & & $0,021^{*}$ & $0,000^{*}$ & 0,196 & 0,524 \\
REC & 0,761 & 0,492 & 0,566 & 0,116 & & 0,221 & 0,197 & 0,177 \\
RJ & $0,041^{*}$ & 0,081 & 0,059 & $0,000^{*}$ & 0,770 & & 0,897 & $0,003^{*}$ \\
SAL & 0,426 & 0,714 & 0,889 & 0,289 & 0,300 & 0,237 & & 0,313 \\
SP & $0,025^{*}$ & $0,004^{*}$ & 0,909 & 0,895 & 0,076 & $0,000^{*}$ & 0,146 & \\
\hline
\end{tabular}

Fonte: Elaborado pelos autores. Teste de causalidade com uma defasagem. (b) Tabela reporta o p-valor do teste. *Significância de $5 \%$.

Tabela A.7: Causalidade de Granger entre os ciclos individuais e comuns (modelo restrito) (b)

\begin{tabular}{lcccccccc}
\hline Causalidade & BH & CUR & FOR & POA & REC & RJ & SAL & SP \\
\hline C. Comum \#1 >C. Individual & 0,422 & 0,398 & 0,877 & 0,417 & 0,371 & 0,128 & 0,289 & 0,206 \\
C. Individual >C. Comum \#1 & 0,087 & 0,141 & 0,280 & 0,101 & 0,394 & 0,217 & 0,531 & 0,147 \\
C. Comum \#7 >C. Individual & 0,551 & 0,222 & 0,681 & 0,692 & 0,519 & 0,604 & 0,904 & 0,607 \\
C. Individual >C. Comum \#7 & 0,297 & 0,937 & 0,131 & 0,529 & 0,067 & 0,604 & 0,059 & 0,749
\end{tabular}

Fonte: Elaborado pelos autores. Teste de causalidade com uma defasagem. (b) Tabela reporta o p-valor do teste. ${ }^{*}$ Significância de $5 \%$. 